\title{
Longitudinal evolution of magnetization of ferromagnets following ultrafast demagnetization: Role of finite size and shape of demagnetized region
}

\author{
I.A. Yastremsky \\ Taras Shevchenko National University of Kyiv, 64 Volodymyrska St., 01601 Kyiv, Ukraine \\ Received September 4, 2014, in final form October 8, 2014
}

A dependence of a relaxation rate on the shape of the demagnetized region for a longitudinal evolution of total magnetization to its equilibrium value following the ultrafast demagnetization is demonstrated. This shapedependence is caused by a motion of the wave front inside the demagnetized region. The contribution of the wave front for spherically symmetric shape of the demagnetized region is up to 3 times and for cylindrically symmetric shape up to 2 times stronger than for one dimensional demagnetized region. This effect can be observed after the demagnetization by a tightly focused femtosecond laser pulse.

Key words: ultrafast demagnetization, ferromagnets, femtosecond laser

PACS: 75.78.Jp, 78.47.J

\section{Introduction}

Recently, in the works where for a tightly focused (up to $1 \mu \mathrm{m}$ ) femtosecond laser pulse it was possible to observe the real picture of the spatial-temporal evolution under the action of a solitary pulse, the authors reported on the development of strongly inhomogeneous states. Along with the propagation of spin waves far away from the light spot [1 2], the authors observed the formation of domains [3] and ring structures localized near the spot [2]. Strongly inhomogeneous distributions of the magnetization appear in the framework of superdiffusive mechanism of action of the laser pulse on magnetic metals [4] 5] and the existence of these distributions is established for magnetic heterostructures [6 7]. Thus, the analysis of the picosecond, longitudinal evolution of inhomogeneous, nonequilibrium distributions of magnetization has become increasingly important.

The analysis of such states can be performed using the Landau-Lifshitz equation with a relaxation term proposed by Bar'yakhtar [8-10], also referred to as LLBar equations [11]. LLBar equations were derived using general principles (Onsager's relations, symmetry of the exchange interaction), and they have no alternatives for the study of the highly non-linear, nonequilibrium, non-uniform states with strong reduction of magnetization mentioned above. LLBar equations are well suited for a description of non-uniform states, such as magnetic solitons [12, 13] and Bloch points [14], and give the explanation of the reversal effects [15 16]. These equations provide an explanation [7] of recent experiments [6] on magnetization recovery in laser-pumped $\mathrm{Ni}-\mathrm{Ru}$-Fe heterostructures, where the importance of the nonlocal character of the magnetization recovery is established [7].

A longitudinal relaxation of a total magnetization of ferromagnets following the ultrafast demagnetization within one dimensional (1d) model is determined by two effects: a homogeneous evolution of a magnetization inside the demagnetized region and the motion of the wave front from the outside to within the demagnetized region [17]. For a sufficiently large diameter of the demagnetized region, a homogeneous evolution dominates. However, for a smaller demagnetized region, a relative contribution of 
the wave front increases and for tightly focused femtosecond laser pulse the motion of the wave front can significantly enhance the relaxation [17, 18].

In this paper, the effect of the size and shape of the demagnetized region on the relaxation rate of the total magnetization is analyzed. In order to demonstrate this effect, we consider the nonlinear, longitudinal evolution of the total magnetization following the ultrafast demagnetization for two limiting cases, namely, for the demagnetized region of cylindrical (CS) and spherical (SS) symmetries. Then, we compare these results with the results found within $1 \mathrm{~d}$ model.

A strongly non-equilibrium state created by the laser pulse plays the role of the initial condition for LLBar equations [7]. For our case, since the motion of the wave front is associated with the change in the total magnetization of the ferromagnet, and the size of a transition region between demagnetized and non-perturbed regions is much larger than nanometers, the exchange (nonlocal) term, which retains the total magnetization of a sample, can be disregarded (compare with [7 15]) and the main contribution to the equations of motion is determined by relativistic processes. Since during the relaxation of magnetization toward an equilibrium value the effective field is parallel to the magnetization, only the modulus of the magnetization $M=|\mathbf{M}|$ enters the equation. Adopting the Landau model for the free energy and introducing dimensionless variables, the nonlinear diffusion equation (NDE) is derived for the evolution of $M[7,18]$

$$
\frac{\partial m}{\partial \tau}=\nabla^{2} m+m\left(1-m^{2}\right)
$$

where $m(\xi, \tau)=M / M_{0}$ is a dimensionless magnetization, $M_{0}$ is an equilibrium value (temperaturedependent) of the magnetization of a bulk material, $\xi$ is a dimensionless radius in cylindrical or spherical coordinates measured in units of $r_{0}=\sqrt{2 A \chi_{\|}}$(as we consider cylindrically and spherically symmetric problems, only $\xi$ enters the problem), $A$ is an inhomogeneous exchange constant, $\chi_{\|}=\mathrm{d} M / \mathrm{d} H$ is a longitudinal magnetic susceptibility of a material in the equilibrium state and at zero magnetic field and $\tau$ is a dimensionless time measured in units of $t_{0}=2 \chi_{\|} / \gamma \lambda_{\mathrm{r}} M_{\mathrm{S}}, \gamma$ is the gyromagnetic ratio, $\lambda_{\mathrm{r}}$ is a dimensionless relaxation constant of the relativistic nature, $M_{\mathrm{S}}$ is the saturation magnetization. Simple estimates for nickel show that the value $r_{0}$ is of the order of a lattice constant, the characteristic time $t_{0}$ is of the order of a few picoseconds and the characteristic velocity $r_{0} / t_{0} \approx 0.1 \mathrm{~nm} / \mathrm{ps}=100 \mathrm{~m} / \mathrm{s}$, see for details [18]. Note that the use of the continuum approximation for distributions with characteristic sizes of the order of the lattice constant does not lead to qualitative errors, which can be seen from comparison of the results obtained numerically for discrete models and for their continual counterpart, see, e.g., [19]20]. It is worth noting that this kind of NDE was first studied in the pioneering work by Kolmogorov, Petrovsky and Piskunov [21] and by Fisher [22] and stationary diffusive front propagations into unstable state have been found for this problem.

After the pulse action, the demagnetized region (spot) is formed in a sample with a characteristic size of the order of a diameter of the laser beam. The value of magnetization is reduced inside the spot, $m=m_{0}<1$, and outside the spot the magnet is non-perturbed, $m=1$. We consider two cases, when the demagnetized region has a cylindrical and spherical symmetries. To model such a situation, NDE is numerically solved for the following initial conditions

$$
m(\xi, \tau=0)=m_{0}+\frac{1-m_{0}}{1+\exp \left[-4\left(\xi-R_{0}\right) / a\right]},
$$

where $R_{0}$ is the radius of the demagnetized region, a parameter $a=\left.\left(1-m_{0}\right)(\mathrm{d} m / \mathrm{d} \xi)^{-1}\right|_{m=\left(1+m_{0}\right) / 2}$ describes the characteristic width of the transition region in the initial conditions. In the region of the action of the laser pulse $\left(\xi<R_{0}\right)$, the magnetization tends to $m_{0}$ and outside this region $\left(\xi>R_{0}\right) m(\xi, \tau=0)$ tends to its equilibrium value 1.

For a numerical analysis we consider the limiting case, when the laser pulse is focused to a diffraction limited spot of the order of $1 \mu \mathrm{m}$ [2]. The following values of the parameters (in the dimensionless units) correspond to the following situation: a radius of the demagnetized region (laser pump) $R_{0}=1250$, the width of transition region $a=300$ and the radius of the sample $R=1850$. We consider the evolution of the initial state 1.2 for the following minimal values of magnetization in the demagnetized region: $m_{0}=0.9,0.5,10^{-2}, 10^{-4}, 0$. The values $m_{0}=0.5,0.9$ can be realized for a weak intensity of the laser pulse and correspond to the effective temperature of the spin system lower than the Curie temperature $T_{\mathrm{C}}$. The values $m_{0}=10^{-2}, 10^{-4}, 0$ can be realized for a high power of the laser pulse. 


\section{Cylindrically symmetric initial distribution of magnetization}

Figure 1 (a) presents the time evolution of the relative change of magnetization per unit length of the sample $M_{\Sigma}(\tau)$

$$
\frac{\Delta M_{\Sigma}(\tau)}{\Delta M_{\Sigma}}=\frac{M_{\Sigma}(\tau)-M_{\Sigma}(0)}{M_{\Sigma}(+\infty)-M_{\Sigma}(0)},
$$

calculated in the time domain from $\tau=0$ to $\tau=T=15$ (in the dimensionless units $t_{0}$ ), for the above chosen parameters and for the initial conditions 1.2. Figure 1 (b) demonstrates an example of the corresponding evolution of magnetization $m(\xi, \tau)$ on the time for $m_{0}=10^{-2}$.

Figure 1 (a) shows that the fastest regime of relaxation of the total magnetization to its equilibrium value is realized for $m_{0}=0.9$. The relaxation time of $\Delta M_{\Sigma}(\tau) / \Delta M_{\Sigma}$, as for $1 \mathrm{~d}$ model [17], decays with a decrease of $m_{0}$ and takes its minimum value at $m_{0}=0$. However, the dependence of $\Delta M_{\Sigma}(\tau) / \Delta M_{\Sigma}$ on time is not linear for $m_{0}=0$ in CS case. The analysis of the evolution of $m(\xi, \tau)$ [figure 1](b)] demonstrates that similarly to $1 \mathrm{~d}$ model [17, 18], the evolution of the total magnetization to its equilibrium value is determined by two effects: the homogeneous evolution of the magnetization and the motion of the wave front within the demagnetized region.

Let us derive an approximate analytical formula for the evolution of $M_{\Sigma}(\tau)$ for CS case, which takes into account both the motion of the wave front and the homogeneous evolution of the magnetization and estimate the contribution of the wave front compared to $1 \mathrm{~d}$ model. Following [17], the evolution of the wave front can be cast in the form

$$
m(\xi, \tau) \approx m_{0}(\tau)+\left[1-m_{0}(\tau)\right] m_{\mathrm{f}}\left(\xi-\xi_{0}-V \tau, \xi\right),
$$

where $m_{0}(\tau)$ does not depend on the coordinate and describes the homogeneous evolution of magnetization within the demagnetized region. The second term in the right-hand side of (2.2) corresponds to the wave front, which moves within the demagnetized region with an amplitude $1-m_{0}(\tau)$ and the velocity $V$. The function $m_{\mathrm{f}}$ describes the shape of this front; the value of $m_{\mathrm{f}}$ tends to 1 if one moves outside the demagnetized region from the wave front and $m_{\mathrm{f}}$ tends to 0 within the demagnetized region. Both $V$ and the shape of the wave front depend on the distance from the center of the demagnetized region $\xi$. However, this problem can be simplified by the following observation: figure 1 (b) shows that the contribution of the wave front is essential only at the initial stage of the evolution. This means that: (i) one can neglect the term $(1 / \xi) \partial m / \partial \xi \propto 1 /(a \cdot \xi) \ll 1 / a^{2}$ and 1.1 transforms to $1 \mathrm{~d}$ NDE; (ii) we can suppose that within the demagnetized region $m(\xi, \tau) \simeq m_{0}(\tau) \ll 1$ and the terms of the order of $m_{0}(\tau) \ll 1$ can be neglected in this approximation. Then, the shape $m_{\mathrm{f}}$ coincides with the shape of the wave front at $m_{0}=0$ in $1 \mathrm{~d}$

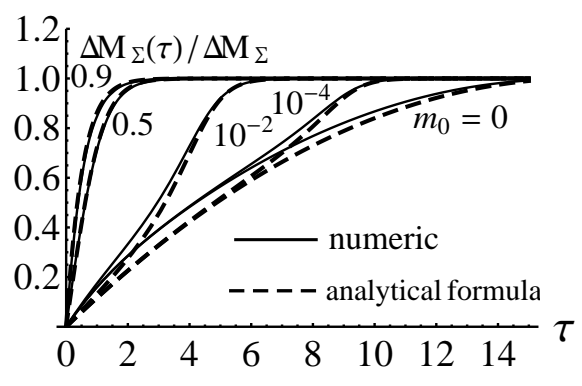

(a)

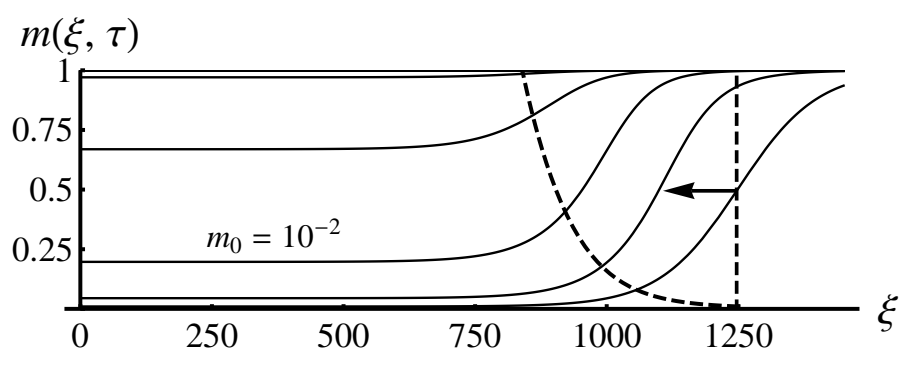

(b)

Figure 1. (a) The time evolution of $\Delta M_{\Sigma}(\tau) / \Delta M_{\Sigma}$, derived from the numerical solution of NDE for the above chosen numerical parameters, different values of $m_{0}$ and for CS initial conditions. Full lines represent numerical calculations and dashed lines are an approximation with the analytical equation [2.4. (b) A corresponding to figure 1 (a) evolution of the magnetization $m(\xi, t)$ for $m_{0}=10^{-2}$ on a time domain from $\tau=0$ to $\tau=T=15$, taken step-by step after an interval of time $T / 10$. Dashed lines show the contributions of the wave front and the homogeneous evolution of magnetization. The arrow indicates a direction of motion of the wave front. 
NDE and the velocity of the wave front $V$ formed from the initial conditions can be found by equating the asymptotic of (1.2) within the demagnetized region and that of the wave front: $V=a / 4+4 / a$ [23]. For the chosen numerical parameters $V \approx 75.01$. Neglecting dispersion in (1.1) and integrating this equation, the dependence of the magnetization on time $m_{0}(\tau)$ within the demagnetized region can be present as follows [7]:

$$
m_{0}(\tau)=\frac{m_{0}}{\sqrt{m_{0}^{2}+\left(1-m_{0}^{2}\right) \exp (-2 \tau)}} .
$$

Thus, at $m_{0}(\tau) \ll 1$ the homogeneous evolution of magnetization leads to a decrease of the amplitude of the wave front $\left[1-m_{0}(\tau)\right]$, and the motion of the wave front leads a decrease of the characteristic radius of the demagnetized region, which is equal to $\left(R_{0}-V \tau\right)$.

Integrating (2.2) over the unit length of the cylinder, the estimating formula for the evolution of $M_{\Sigma}(\tau)$ is derived

$$
M_{\Sigma}(\tau) \approx M_{\Sigma}(0)+\pi R_{0}^{2} m_{0}(\tau)+\pi\left[1-m_{0}(\tau)\right]\left(2 R_{0}-V \tau\right) V \tau,
$$

where $m_{0}(\tau)$ is defined by (2.3). Equation (2.4) takes into account both the homogeneous evolution of magnetization and the motion of the wave front.

Figure 1 (a) shows a good agreement between the analytical equation (2.4) and the numerical results even in the regime, at which the magnetization within the demagnetized region $m_{0}(\tau)$ is not small, when both the shape and the velocity of the wave front depend on $m_{0}(\tau)$. The reason here is that for this regime the contribution of the wave front becomes small.

Let us estimate the relative contribution of the wave front and the homogeneous evolution of magnetization for different $m_{0}$ for CS case. We present

$$
\frac{\mathrm{d} M_{\Sigma}(\tau)}{\mathrm{d} \tau}=\frac{\mathrm{d} M_{\Sigma}^{\text {wave }}(\tau)}{\mathrm{d} \tau}+\frac{\mathrm{d} M_{\Sigma}^{\text {flat }}(\tau)}{\mathrm{d} \tau},
$$

where $M_{\Sigma}^{\text {wave }}(\tau)$ and $M_{\Sigma}^{\text {flat }}(\tau)$ are contributions of the wave front and the homogeneous evolution, respectively [these contributions are schematically depicted in figure 1 (b) by dashed lines]. Differentiating 2.4) with respect to time and comparing the result with [2.5), one can see that

$$
\frac{\mathrm{d} M_{\Sigma}^{\text {wave }}}{\mathrm{d} \tau} \simeq 2 \pi\left(R_{0}-V \tau\right)\left[1-m_{0}(\tau)\right] V, \quad \frac{\mathrm{d} M_{\Sigma}^{\text {flat }}}{\mathrm{d} \tau} \simeq \pi\left(R_{0}-V \tau\right)^{2}\left[m_{0}(\tau)-m_{0}(\tau)^{3}\right] .
$$

Here, $\left[1-m_{0}(\tau)\right]$ is the amplitude of the wave front, $\left(R_{0}-V \tau\right)$ is the characteristic radius of the demagnetized region at the moment $\tau$.

Thus, $\mathrm{d} M_{\Sigma}^{\text {wave }}(\tau) / \mathrm{d} \tau$ takes its maximum value at the initial stage of the evolution. However, further, the the relaxation rate caused by the wave front decreases with a decrease of both the amplitude and the length of the wave front.

The relative contribution of the wave front can be defined as $M_{\Sigma}^{\text {wave }}(\tau) / \Delta M_{\Sigma}$ at $\tau \rightarrow+\infty$, where $M_{\Sigma}^{\text {wave }}(\tau)$ can be found by integrating (2.6). Table 1 presents $M_{\Sigma}^{\text {wave }}(\tau) / \Delta M_{\Sigma}$ for different $m_{0}$, for $1 \mathrm{~d}$ model [17], CS and SS cases. Table 1 demonstrates that the contribution of the wave front for CS is stronger than for $1 \mathrm{~d}$ model (almost two times stronger for not small $m_{0}$ ). To understand this observation one should note that the wave front gives the main contribution at the initial stage. Then, estimating $\mathrm{d} M_{\Sigma}^{\text {wave }} /\left(\Delta M_{\Sigma} \mathrm{d} \tau\right)$ at $\tau=0$ (initial stage), we see that this value is two times larger in CS case than that found within $1 \mathrm{~d}$ model. With a decrease of $m_{0}, M_{\Sigma}^{\text {wave }} / \Delta M_{\Sigma}$ increases and takes its maximum value 1 for $m_{0}=0$.

\begin{tabular}{|l|l|l|l|l|}
\hline \hline$m_{0}$ & 0.5 & $10^{-2}$ & $10^{-4}$ & 0 \\
\hline$M_{\Sigma}^{\text {wave }} / \Delta M_{\Sigma}(1 \mathrm{~d})$ & 0.05 & 0.24 & 0.51 & 1 \\
\hline$M_{\Sigma}^{\text {wave }} / \Delta M_{\Sigma}(\mathrm{CS})$ & 0.09 & 0.41 & 0.75 & 1 \\
\hline$M_{\Sigma}^{\text {wave }} / \Delta M_{\Sigma}(\mathrm{SS})$ & 0.13 & 0.53 & 0.85 & 1 \\
\hline \hline
\end{tabular}

Table 1. The relative contribution of the wave front for $1 \mathrm{~d}, \mathrm{CS}$ and SS cases for the above chosen values of the parameters. 
To estimate the value of magnetization in the demagnetized region $m_{\mathrm{eq}}^{\mathrm{CS}}$ (for CS case), for which the relaxation rates for the wave front and the homogeneous evolution become comparable, we equate $\mathrm{d} M_{\Sigma}^{\text {wave }} / \mathrm{d} \tau=\mathrm{d} M_{\Sigma}^{\text {flat }} / \mathrm{d} \tau$. This is a nonlinear, transcendental equation. However, a simple estimating formula can be derived by noting that the wave front gives the main contribution at the initial stage, that is $m_{\mathrm{eq}}^{\mathrm{CS}} \ll 1$. Then, neglecting the terms like $\left(m_{\mathrm{eq}}^{\mathrm{CS}}\right)^{2}$, we get

$$
m_{\mathrm{eq}}^{\mathrm{CS}} \simeq 2 V / R_{0} .
$$

Thus, if the initial value of magnetization in the demagnetized region for CS situation $m_{0} \ll m_{\mathrm{eq}}^{\mathrm{CS}}$, the wave front gives a substantial contribution to the relaxation of the total magnetization. Note, that $m_{\mathrm{eq}}^{\mathrm{CS}}$ is two times larger than for $1 \mathrm{~d}$ model $m_{\mathrm{eq}}^{1 \mathrm{~d}} \simeq V / R_{0}$ [17.

\section{Spherically symmetric initial distribution of magnetization}

The evolution of the wave front for SS case can be also presented in the form (2.2). Using the assumptions similar to the CS case and integrating (2.2) over the volume of the sample, the estimating formulae are derived

$$
M_{\Sigma}(\tau) \approx M_{\Sigma}(0)+\frac{4 \pi}{3} R_{0}^{3} m_{0}(\tau)+4 \pi V \tau\left[1-m_{0}(\tau)\right]\left(R_{0}^{2}-R_{0} V \tau+\frac{V^{2} \tau^{2}}{3}\right),
$$

where $m_{0}(\tau)$ is also defined by (2.3), $M_{\Sigma}(\tau)$ is the total magnetization of the sample and

$$
\frac{\mathrm{d} M_{\Sigma}^{\text {wave }}}{\mathrm{d} \tau} \simeq 4 \pi\left(R_{0}-V \tau\right)^{2}\left[1-m_{0}(\tau)\right] V, \quad \frac{\mathrm{d} M_{\Sigma}^{\text {flat }}}{\mathrm{d} \tau} \simeq \frac{4 \pi}{3}\left(R_{0}-V \tau\right)^{3}\left[m_{0}(\tau)-m_{0}(\tau)^{3}\right] .
$$

These formulae well describe the results of the numerical calculations. Table 1 demonstrates that the contribution of the wave front for SS case is stronger than for CS case (almost 1.5 times for not small $m_{0}$ ). Estimating $\mathrm{d} M_{\Sigma}^{\text {wave }} /\left(\Delta M_{\Sigma} \mathrm{d} \tau\right)$ at the initial stage, we see that $\mathrm{d} M_{\Sigma}^{\text {wave }} /\left(\Delta M_{\Sigma} \mathrm{d} \tau\right)$ at $\tau=0$ for SS case is 1.5 times larger than in the CS case, thus giving the explanation of the numerical data. Comparing different contributions in 3.2 , we get

$$
m_{\mathrm{eq}}^{\mathrm{SS}} \simeq 3 V / R_{0} .
$$

So, $m_{\mathrm{eq}}^{\mathrm{SS}}$ is 1.5 times larger than for the CS case.

\section{Conclusion}

The relaxation of highly nonequilibrium, nonuniform states created by the femtosecond laser pulse in the relativistic relaxation approximation is governed by two scenarios: the motion of the wave front within the demagnetized region and the homogeneous evolution of magnetization within the demagnetized region. The relativistic relaxation approximation is valid for widths of the transition regions between the demagnetized and non-perturbed regions exceeding a few dozens nanometers (compare with [7]). The contribution of the wave front for the SS shape of the demagnetized region is up to 3 times and for CS case is up to 2 times stronger than for $1 \mathrm{~d}$ model [17]; thus, the motion of the wave front within the demagnetized region leads to the dependence of the relaxation rate on the shape of the demagnetized region. For a sufficiently large diameter of the demagnetized region (laser spot), the homogeneous evolution dominates and the evolution of the total magnetization is the same for $1 \mathrm{~d}$ model, CS and SS cases. However, with a decrease of the diameter of the demagnetized region and with an increase of the power of the laser impulse, the relative contribution of the wave front increases and for $m_{0}<\left(m_{\mathrm{eq}}^{\mathrm{CS}}, m_{\mathrm{eq}}^{\mathrm{SS}}\right)$ the wave front can significantly enhance the relaxation and, consequently, the shape dependent effects can also be observed. This situation could be realized after demagnetization by a tightly focused femtosecond laser impulse.

\section{Acknowledgements}

I thank prof. B.A. Ivanov for the help in the formulation of the problem and for fruitful discussions. 


\title{
References
}

1. Satoh T., Terui Y., Moriya R., Ivanov B.A., Ando K., Saitoh E., Shimura T., Kuroda K., Nat. Photonics, 2012, 6, No. 10, 662; doi 10.1038/NPHOTON.2012.218

2. Au Y., Dvornik M., Davison T., Ahmad E., Keatley P.S., Vansteenkiste A., Van Waeyenberge B., Kruglyak V.V., Phys. Rev. Lett., 2013, 110, 097201; doi 10.1103/PhysRevLett.110.097201.

3. De Jong J.A., Razdolski I., Kalashnikova A.M., Pisarev R.V., Balbashov A.M., Kirilyuk A., Rasing Th., Kimel A.V., Phys. Rev. Lett., 2012, 108, 157601; doi 10.1103/PhysRevLett.108.157601

4. Battiato M., Carva K., Oppeneer P.M., Phys. Rev. Lett., 2010, 105, 027203; doi 10.1103/PhysRevLett.105.027203

5. Battiato M., Carva K., Oppeneer P.M., Phys. Rev. B, 2012, 86, 024404; doi 10.1103/PhysRevB.86.024404

6. Rudolf D., La-O-Vorakiat C., Battiato M., Adam R., Shaw J.M., Turgut E., Maldonado P., Mathias S., Grychtol P., Nembach H.T., Silva T.J., Aeschlimann M., Kapteyn H.C., Murnane M.M., Schneider C.M., Oppeneer P.M., Nat. Commun., 2012, 3, 1037; doi 10.1038/ncomms2029

7. Yastremsky I.A., Oppeneer P.M., Ivanov B.A., Phys. Rev. B, 2014, 90, 022409; doi 10.1103/PhysRevB.90.024409

8. Bar'yakhtar V.G., Zh. Eksp. Theor. Fiz., 1984, 87, 1501 (in Russian) [Sov. Phys. JETP, 1984, 60, 863].

9. Bar’yakhtar V.G., Fiz. Tverd. Tela, 1987, 29, 1317 (in Russian) [Sov. Phys. Solid State, 1987, 29, 754].

10. Bar’yakhtar V.G., Physica B, 1989, 159, 20; doi 10.1016/S0921-4526(89)80047-X

11. Dvornik M., Vansteenkiste A., Van Waeyenberge B., Phys. Rev. B, 2013, 88, 054427; doi 10.1103/PhysRevB.88.054427

12. Bar’yakhtar V.G., Ivanov B.A., Sobolyeva T.K., Sukstansky A.L., Zh. Eksp. Teor. Fiz., 1986, 91, No. 4, 1454 (in Russian) [Sov. Phys. JETP, 1986, 64, No. 4, 857].

13. Bar’yakhtar V.G., Ivanov B.A., Safaryan K.A., Solid State Commun., 1989, 72, 1117; doi 10.1016/0038-1098(89)90257-3

14. Galkina E.G., Ivanov B.A., Stephanovich V.A., J. Magn. Magn. Mater., 1993, 118, No. 3, 373; doi 10.1016/03048853(93)90441-4

15. Mentink J.H., Hellsvik J., Afanasiev D.V., Ivanov B.A., Kirilyuk A., Kimel A.V., Eriksson O., Katsnelson M.I., Rasing Th., Phys. Rev. Lett., 2012, 108, 057202; doi 10.1103/PhysRevLett.108.057202

16. Bar’yakhtar V.G., Butrim V.I., Ivanov B.A., JETP Lett., 2013, 98, No. 5, 289; doi 10.1134/S0021364013180057.

17. Yastremsky I.A., Fiz. Nizk. Temp., 2014, 40, No. 11, 1251 (in Russian) [Low Temp. Phys., 2014, 40, 978; doi $10.1063 / 1.4903339]$.

18. Yastremsky I.A., Fiz. Tverd. Tela, 2014, 56, 1076 (in Russian) [Phys. Solid State, 2014, 56, No. 6, 1118; doi $10.1134 /$ S1063783414060390].

19. Ivanov B.A., Kolezhuk A.K., Wysin G.M., Phys. Rev. Lett., 1996, 76, No. 3, 511; doi 10.1103/PhysRevLett.76.511

20. Ivanov B.A., Wysin G.M., Phys. Rev. B, 2002, 65, 134434; doi 10.1103/PhysRevB.65.134434

21. Kolmogorov A., Petrovsky I., Piskunov N., In: Dynamics of Curved Fronts, Pelcé P. (Ed.), Academic Press, Boston, 1988, 105-130.

22. Fischer R., Ann. Eugenics, 1937, 7, 355; doi 10.1111/j.1469-1809.1937.tb02153.x

23. Kametaka Y., Osaka J. Math., 1976, 13, 11.

\section{Поздовжня еволюція намагніченості феромагнетика після надшвидкого розмагнічування: роль розміру та форми розмагніченої області}

\author{
I.О. Ястремський \\ Київський національний університет імені Тараса Шевченка, \\ вул. Володимирська, 64, 01601 Київ, Україна
}

\begin{abstract}
Продемонстровано залежність швидкості релаксації поздовжньої еволюції повної намагніченості феромагнетика після надшвидкого розмагнічування від форми розмагніченої області. Ця залежність обумовлена рухом хвильового фронту вглиб розмагніченої області. Внесок хвильового фронту для сферичносиметричної форми розмагніченої області до 3 раз та для циліндрично-симетричної області до 2 раз більший, ніж для одновимірного випадку. Цей ефект може спостерігатись після розмагнічування сильно сфокусованим лазерним імпульсом.
\end{abstract}

Ключові слова: надшвидке розмагнічування, феромагнетик, фемтосекундний лазер 